\title{
Population synthesis from clustered star formation
}

\author{
M. R. Haas ${ }^{1}$ \& P. Anders ${ }^{2}$ \\ ${ }^{1}$ Leiden Observatory, Leiden University \\ Postbus 9513, NL-2300RA, Leiden, the Netherlands \\ email: haas@strw.leidenuniv.nl \\ ${ }^{2}$ Sterrenkundig Instituut, University of Utrecht, \\ email: p.anders@uu.nl
}

\section{Introduction}

In recent years, a series of papers (Kroupa \& Weidner 2003, Weidner \& Kroupa 2004, Weidner \& Kroupa 2005 and Weidner \& Kroupa 2006, WK06 from now on) have proposed that the stellar content of an entire galaxy may not be well described by the same initial mass function (IMF) that describes the distribution of stellar masses in the star clusters, where these stars form. The reason is that star clusters also form with a cluster mass function $(\mathrm{CMF})$, which is a power law with a power law index of $\sim-2$. If the lowest mass clusters are of masses smaller than the physical upper mass limit for stars they will be deficient in high mass stars. Therefore, if the stellar content of all clusters is added together, making up the Integrated Galactic Initial Mass Function (IGIMF), the distribution of stellar masses may be steeper at the high mass end, depending on the exact shape of the CMF.

In most earlier studies, the assumed CMF and sampling method are kept the same. We investigate the dependence of the results on these assumptions and check the observability in terms of stellar content, integrated photometry and chemical enrichment of galaxies.

\section{Several IGIMFs}

We test several sampling methods which are described in detail in Haas \& Anders (2009). We use a Monte Carlo approach in order to make the numerical scatter smaller than the difference between IGIMFs. Tests using either a Salpeter (1955) IMF or a Kroupa (2001) IMF are virtually indistinguishable.

As the main cause of the lack of high mass stars are the low mass clusters, the behaviour of the CMF at the low mass end is very important. We test various CMFs with various power law indices and various lower mass cut-offs.

The different sampling methods give different IGIMFs. Depending on the method, the steep end has power law indices of about -2.6 , as compared to the input -2.35 . The sorted sampling and analytic sampling get even slightly steeper and deviate strongly from a power law shape. The recovered CMFs after filling the clusters with stars are indistinguishable from the input CMF.

From the realisations using different CMFs, it results that the slope of the CMF determines the high mass end slope of the IGIMF, whereas the minimum cluster mass sets the stellar mass at which the steepening sets in. If clusters do not go down in mass all the way to the mass range of stars, sampling effects are negligible. The low mass end of the CMF is badly constrained. The IGIMF could be obtained observationally, giving an indirect measure of the low mass end of the CMF (under the assumption of a sampling method and an underlying IMF). 


\section{Galaxy properties from IGIMFs}

Using the IGIMFs as IMFs in galaxy evolution models we are able to investigate the integrated properties of galaxies, if their total stellar initial mass distribution is given by an IGIMF rather than a more standard IMF.

Assuming that GAIA will observe $10 \%$ of all O-stars in the Galaxy, that they live for 10 Myr and that in the past 10 Myr the average SFR of the Galaxy was $1 \mathrm{M}_{\odot} / \mathrm{yr}$, GAIA will be able to rule out several of the very extreme IGIMFs (from e.g. steep CMFs and sorted sampling) with very high significance. Judging between the several different sampling methods is difficult, as the differences are of the order of $1 \sigma$, for purely poissonian errors on the number counts. Besides, the numbers depend on the exact values of the SFR averaged over $10 \mathrm{Myr}$ and the fraction of all O-stars observed (which depends on their a priori unknown location).

Using the GALEV chemically self-consistent, closed box evolutionary synthesis models (Bicker et al. 2004, Kotulla et al. 2009) we are able to assess observable properties of galaxies, in which the stellar initial mass distribution is given by IGIMFs. The ingredients of the models are star formation histories which depend on the gas mass present (no inand outflows are assumed, nor are they necessary) and Hubble Type, spectral synthesis, and yield tables. For all Hubble types, the difference in integrated photometry in any band is smaller than the observed galaxy-to-galaxy scatter as present in the HyperLeda database $\nmid$ (Paturel et al. 2003). Therefore, integrated photometry of galaxies will not be a discriminant between IMFs and/or IGIMFs.

With IGIMFs, metallicities of galaxies vary by half a dex at given gas mass fraction of the galaxies. For the IGIMFs resulting from extremely steep CMFs, the difference can be up to 2 orders of magnitude. At given gas mass fractions, the HyperLeda database gives a scatter of about half a dex as well. Therefore, the most extreme CMFs can be ruled out by the observed gas phase metallicities. In order to differentiate between several IGIMFs more precise measurements of gas metallicities and gas fractions are necessary. The difference between sampling methods is comparable to, or slightly larger than the spread that results from using individual metallicity measurements for the same galaxy, and is thus a possible discriminant between IGIMFs, provided that absolute determinations of gas phase metallicities and gas fractions are reliable.

\section{References}

Bicker, J., Fritze-v. Alvensleben, U., Möller, C. S., \& Fricke, K. J. 2004, A\& A, 413, 37

Goodwin, S. P. \& Pagel, B. E. J. 2005, MNRAS, 359, 707

Haas, M. R. \& Anders, P. 2009, A\&\&A, submitted

Kotulla, R., Fritze, U., Weilbacher, P., \& Anders, P. 2009, MNRAS, 396, 462

Kroupa, P. 2001, MNRAS, 322, 231

Kroupa, P. \& Weidner, C 2003, ApJ, 598, 1076

Paturel, G., Petit, C., Prugniel, P., Theureau, G., Rousseau, J., Brouty, M., Dubois, P., \& Cambrésy, L. 2003, A\&\&A, 412, 45

Pflamm-Altenburg, J. \& Kroupa, P. 2008, Nature, 455, 641

Recchi, S., Calura, F., \& Kroupa, P. 2009, A\&A, 499, 711

Salpeter, E. E. 1955, ApJ, 121, 161

Weidner, C. \& Kroupa, P. 2004, MNRAS, 348, 187

Weidner, C. \& Kroupa, P. 2005, ApJ, 625, 754

Weidner, C. \& Kroupa, P. 2006, MNRAS, 365, 1333

$\dagger$ http://leda.univ-lyon1.fr/ 